\title{
Aspects of User Profiling in Computer-based Health Information Tailoring Systems: A Narrative Review
}

\author{
Type of article: conference abstract
}

\author{
Azadeh Kamel Ghalibaf1 ; Zahra Mazloum Khorasani2; Mahdi Gholian Aval3; Mahmood \\ Tara4* \\ 1 Student Research Committee, Department of Medical Informatics, School of Medicine, \\ Mashhad University of Medical Sciences, Mashhad, Iran \\ 2Assistant professor of Endocrinology, Endocrine Research Center, Mashhad University \\ of Medical Sciences, Mashhad, Iran \\ 3Assistant professor, Department of Health Education and Health Promotion, School of \\ Health, Mashhad University of Medical Sciences, Mashhad, Iran. \\ 4Assistant professor, Department of Medical Informatics, School of Medicine, Mashhad \\ University of Medical Sciences, Mashhad, Iran \\ * Phone: 98 5138002429Fax: 98 5138002445Email: taram@mums.ac.ir
}

\begin{abstract}
Introduction:The recent shift from the conventional physician-centered approach to the more polpular approach that with the focuse on patient as the center of healthcare, emphaizes on the critical role of informing and educating patients. Studies shown that tailoring health information to the needs of individuals is more effective than generic materials. Recent improvements in the fields of computer science and Information Communication Technology have made it possible to computerize such an adaptation process. Information tailoring systems use an internal representation of user conditions and needs, which is referred to as a "user model" or "user profile."

A user profile represents the system's beliefs about the user. Hence, it may simply contain demographic information or sophisticated factors such as the state of the disease, user's attitude, interest, preference, and knowledge. The user profile is known as the basis for designing other system components and has a great impact on the acceptance of the system by the user and the quality of the tailored information.

To the best of our knowledge, no studies have been conducted so far to analyze and classify user profile aspects and characteristics. In this systematic narrative review, we aim to provide aspects of profiling in health information tailoring systems based on literature from different diciplines.

Methods:comprehensive searches of the PubMed and Scopus databases have been conducted. We searched among English papers with publishing dates ranging from 1990 onward; since that is when computer-tailoring first appeared within the literature. we have devised a list of terms pertinent to the main concepts of computer-tailoring and used a qualitative-interpretive approach for data extraction.

Results:Analyzing the data from 32 eligible studies, we found three aspects in designing a tailoring user profile. Each aspect with its characteristics are provided below:

1-Identifying common factors used in profiles and classifying these factors thematically, which has three attributes:

a) The number of factors used to design the user profile and their diversity (e.g. demographic,clinical,behavioral information, learning style and so forth)

b) The approaches used to Identify effective factors in tailoring (e.g. evidence-based, avalible data sources)

c) Attributes of the factors (e.g. long-term/short-term, static/dynamic)

2-Data collection tools and methods, which has two attributes:

a) Data collection methods (e.g. explicit, implicit, mixed)

b) Assessment tool (e.g. questionnaire, patient record)

3-Data interpretation that demonstrates to what extent the collected data needs to be analyzed to use in tailoring.
\end{abstract}


we have also identified two main approaches regarding tailoring: public health and computational tailoring. Public Health communication researcher has relied greatly on health behavior models but generally has used simpler technological approaches, whereas computer science employed more advanced technological approaches but integrated behavior theory to a lesser extent. These two approaches complete each other to provide the necessary requirements for designing a practical tailoring system in future studies.

Conclusion: In this study we investigate different aspects of designing a user profile in health information tailoring systems. The proposed model is a valuable guide for new researchers in the field. Results from this review provide a comprehensive overview of the field and will help researchers to combine effective methods from across the disciplines in future research.

Keywords: Health informatics, information tailoring, computer systems, user profile

\section{Declaration of conflicts}

This abstract is selected from the First International Congress of Diseases and Health Outcomes Registry and First National Congress of Medical Informatics, 14-17 February 2017, Mashhad, Iran

\section{Authors' biography}

No biography.

\section{References}

No references. 